\title{
User Log Based Image Re-ranking and Retrieval
}

\author{
S. Sangeetha ${ }^{1}$ and $S$. Varma ${ }^{2}$ \\ ${ }^{1}$ Department of Computer Engineering, PCE, New Panvel \\ ${ }^{2}$ Department of Information Technology, PCE, New Panvel \\ \{s_geetharaj@yahoo.com;vsat2k@yahoo.com\}
}

\begin{abstract}
Digital world has developed tremendously. Initially textual information associated with images is used to do image based search. From user's perspective the final result contains both relevant as well irrelevant images. The expected yield from such a system is to get pertinent result. Satisfaction of the client will be enhanced if re-ranking is used as critical parameter. Images are displayed based on the category selected and reranked according to the query image opted by user. Image re-ranking based on user log technique is used to overcome the issues and to achieve efficient result. Expanding the keyword and clustering is done at offline mode which is also important parameters for the performance of the system. The performance of re-ranked image retrieval is improved with a reduction of time in seconds as compared to existing re-ranking method. The maintained user log helps to produce the relevant search results. The proposed user log system reduces the image retrieval and re-ranking duration from 17 seconds to 4 seconds.
\end{abstract}

Keywords: Text Based Image Retrieval, Re-ranking, Image Retrieval, Clustering, User Log.

\section{Introduction}

The images are retrieved from commercial search engines using text descriptions. The principle issue with such a framework is that the retrieved images have relatively low relevance to user's need. The search performance is improved by reordering the images based on the information extracted from the initial search results and query image. From another point of view, re-ranking can be viewed as a post-process of core search.

Children initial learning is through real objects or pictures. Nowadays every child has their own electronic device through with they try to learn the things. The child enter crane as a query in search engine to know about crane bird. The result is crane machine and crane bird. Now they need to search crane bird among multiple pictures in the collection of crane machine and crane bird which may be difficult. To overcome this issue category based and log based search is provided. The idea is to make school teachers, parents and children use this search tool, for searching images and to know the list of category using simple text. Simple categories according to the level of the children are displayed. Later on the child can see the images without going through all the process with the help of user log.

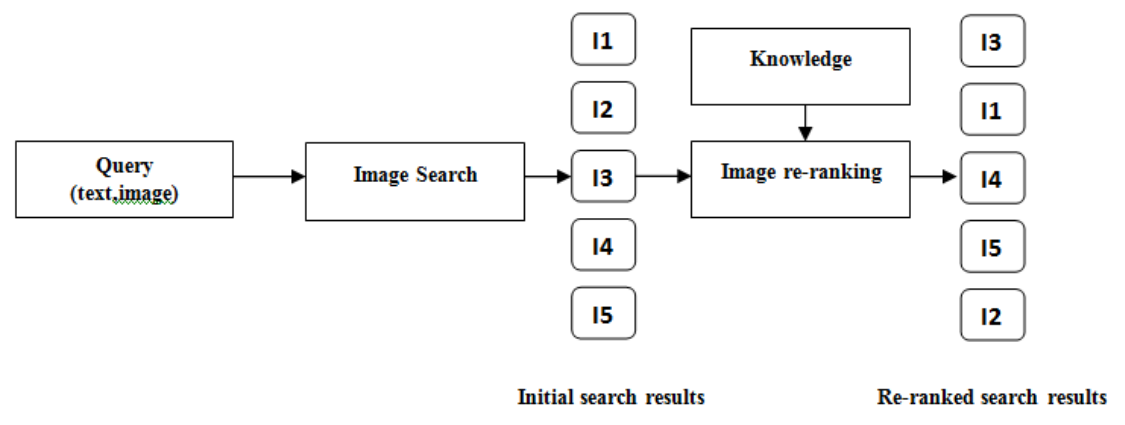

Fig. 1. General image re-ranking process [9]

Fig. 1 explains the general re-ranking process. The input can be text or image based on this the images are displayed according to their ranking score. The images are re-ranked based on other knowledge.

B. Iyer, S. Nalbalwar and R. Pawade (Eds.)

ICCASP/ICMMD-2016. Advances in Intelligent Systems Research.

Vol. 137, Pp. 653-660.

(C) 2017- The authors. Published by Atlantis Press

This is an open access article under the CC BY-NC license (http://creativecommons.org/licens)es/by-nc/4). 


\section{Related Work}

The semantic clustering is proposed by Feng et al. [1]. Semantic clusters are formed based on the given query. Images are moved to corresponding clusters. System results too many similar clusters for the given query.

The Text Based Image Retrieval (TBIR) is based on textual information stored against images. Textual information can be the name of the file, title of the page and tag which is used to identify the images. If the search text is found then images will be retrieved [2].

Xinmei et al. [3] have proposed active re-ranking framework for web based image search. Active re-ranking is based on user interactions. With the knowledge of the user's intention, including both the labeling information and the learned discriminative sub manifold, the re-ranking process is conducted. Several interaction rounds are performed to achieve a satisfactory performance. The active sample selection strategy will reduce the efficiency of the process when users are asked to label the images in much iteration.

The bag-based re-ranking framework for TBIR was proposed by Lixin et al. [4]. Images are moved into clusters based on their textual and visual features. Each cluster is identified as a "bag" and the images in the bag as "instances". The method ranks all the bags according to the defined bag ranking score. It is generally time consuming to manually check a considerable number of bags to obtain one truly positive bag in bag-based re-ranking system.

Xiaoou Tang et al. [5] have used a one click approach. User will select one image as query image and the retrieved images are re-ranked based on their visual similarities. Images are placed into category using adaptive weight schema. Using term frequency and inverted term frequency the top keyword are found for keyword expansion. Images are retrieved for expanded keyword then based on the query image final re-ranked images are displayed. The performance is affected if the initial set of images retrieved contains noisy data.

Jun Huang et al. [8] described Visual Saliency and Consistency algorithm for image re-ranking. The proposed algorithm is to rank the most relevant images first and then to the display the images which are less relevant. The images that closely related to the search query are assumed visually similar. These visually consistent images which occur most frequently in the initial few web pages will be given higher ranks. It is obvious that most noticeable images would be easier to catch users' eyes, and it is observed that these images in the front pages are often relevant to the user's query.

\section{Proposed System}

If Apple is given as query keyword it will retrieve all category related to keyword apple based on text similarity. Images are displayed as per the category selected by the user.

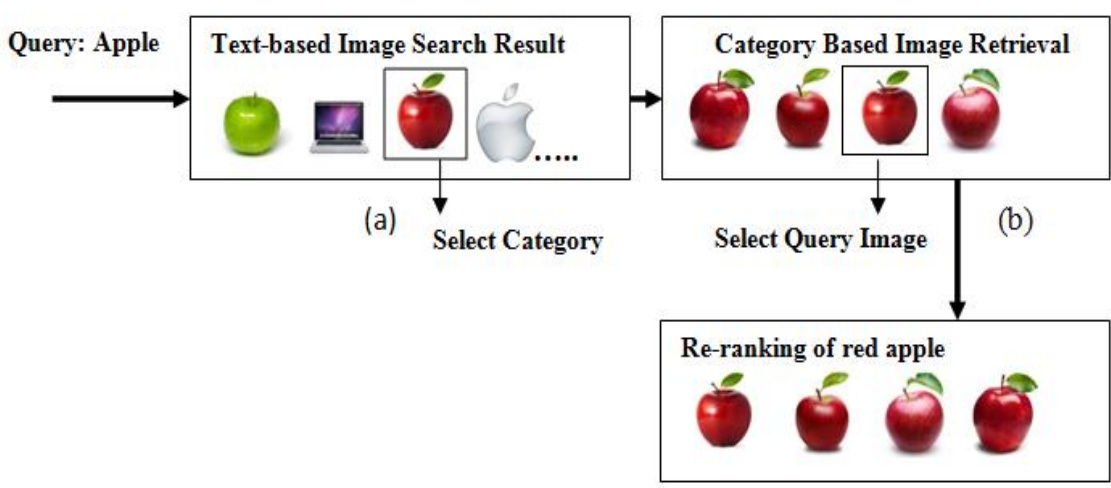

(c)

Fig. 2. Process of Image Re-ranking (a) Images are ranked based on keyword Apple (b) Images are displayed based on category(c) Images re-ranked based on query image

The Fig. 2(a) shows the category based image search result for keyword Apple. For every category the first image is displayed. User need to select a category the user is searching for based on given query keyword. Images are retrieved based on category query image. Fig. 2(b) shows images based category red apple. From the retrieved images single image is selected as query image.

Fig. 2(c) shows the re-ranked image based on query image. The query image of red apple will be stored in database for further search.

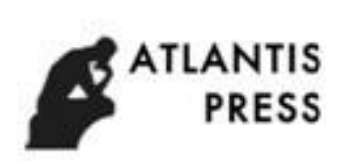


Their applications range from personal use to media, advertising, art, medicine, education and even research in forensic sciences.

\subsection{System Architecture}

The implementation process consists of two phases namely Offline Mode and Online Mode as shown in Fig. 3. Offline Mode is existing method for re-ranking using clustering. Online Mode is proposed technology using user log. In Offline Mode [6], semantic signature is created for every keyword through keyword expansion, clustering and visual features. In online stage, images are re-ranked based on query image and user log from the initial text based search results. Fig. 3 shows the architecture of Image re-ranking using Clustering and User Log.

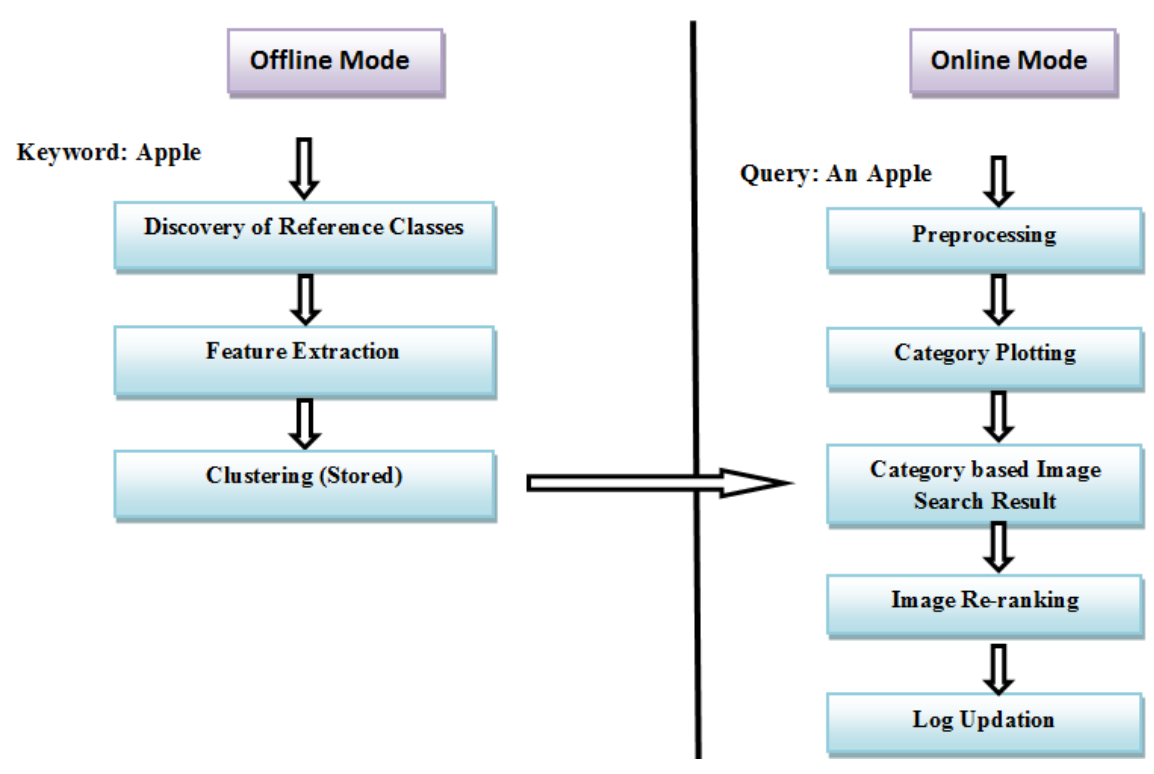

Fig. 3 Architecture of image re-ranking using user log

\section{Algorithm for Offline Mode:}

- Input : Query Keyword in English

- Output : Clustering through keyword expansion

1. Accept the query keyword (q).

2. To create reference classes (Semantic concepts) for each word do

3. Search for image keyword which contains the query keyword

4. Image keyword becomes the expanded keyword

5. For each keyword expansions, retrieve images (I) using the system

6. Low level visual features are extracted for the retrieved set of images and stored

7. For every expanded keyword clustering algorithm is applied on the set of images

8. Clustered data are stored in database

K-means Clustering algorithm: K-means is an unsupervised algorithms used for clustering. The K-means clustered data are stored for further reference. Let $\mathrm{x}$ defines the set of data points and $\mathrm{v}$ defines the set of centre's. Number of clusters consider for offline process are 2.

Step 1: Cluster centers(c) are selected randomly

Step 2: Euclidean distance is used to find the distance between cluster centre and data point. 
Step 3: The minimum distance data point is assign to that cluster out of the entire cluster centre.

Step 4: The new cluster centre is calculated using Equation 1:

$v_{i=}\left(\frac{1}{c_{i}}\right) \sum_{j=1}^{c_{i}} x_{i}$

Where ' $c{ }_{i}$ ' represents the number of data points in $i^{\text {th }}$ cluster.

Step 5: The distance between the new cluster centres and each data point are recalculated.

Step 6: If no data point was reassigned algorithm is not continued, otherwise repeated from step 3.

Online Mode: In online mode search query is entered by user. Pre-processing for the given query is done. During pre-processing stage, stop words are removed. Words which fall under category like pronoun, prepositions and conjunctions are not used during search. They are language specific word which doesn't carry information. If stop words exists then the search is based on all the existing words.eg "an apple" the search are based on word "an" and "apple". So the word "an" is identified as stop word and it is removed. After removing stop word Porter stemming algorithm [11] is used for stemming. After pre-processing the images are retrieved using Text Based Image Retrieval (TBIR). TBIR uses query to retrieve category based images. If there is exact match between keyword and the complete query, images are retrieved for every category. If there is no exact match then the search undergoes text segmentation where every word of the entered query is split into sub query. The search is based on sub query and the images are retrieved and displayed.

The user needs to select a category based on which images are displayed. An image will be selected as query image for the given text names Query Image Based Retrieval (QIBR). The feature of query image is compared with all target images using Equation 2. After comparison the images are arranged according to their ranking score. The query image can be stored by user in user log for further image retrieval. The similarity measurement [12] between two images is defined as follows where $\alpha 1=1$ and $\alpha 2=0$ denote the similarity weighting constants in the image re-ranking system. A small number $\varepsilon=0.1$ is placed at the denominator to avoid the mathematic division error.

$$
\delta(\text { query }, \text { target })=\alpha_{1} \sum_{t=1}^{N_{C}} \frac{\left|C^{\text {query }}(t)-C^{\text {target }}(t)\right|}{C^{\text {query }}(t)+C^{\text {target }}(t)+\varepsilon}+\alpha_{2} \sum_{t=1}^{N_{F}} \frac{\left|F^{\text {query }}(t)-F^{\text {target }}(t)\right|}{F_{\text {query }}(t)+F^{\text {target }}(t)+\varepsilon}
$$

The target image at position 1 has the lowest similarity score, indicating it has the most similar appearance to the query image.

\section{Results and Discussion}

\subsection{Dataset Used}

For experiment purpose 2 datasets are considered for performing the result analysis. First dataset has 1 keyword which has 6 classes and the total number of images used is 250 as shown in Table 1.

Table 1: Description of Dataset1

\begin{tabular}{|c|c|c|c|c|c|c|c|c|}
\hline \multirow{4}{*}{ Dataset1 } & Keyword & Class 1 & Class 2 & Class 3 & Class 4 & Class 5 & Class 6 & \multirow{2}{*}{ Total } \\
\cline { 2 - 8 } & \multirow{2}{*}{ Apple } & $\begin{array}{c}\text { Apple } \\
\text { Iphone }\end{array}$ & $\begin{array}{c}\text { Apple } \\
\text { Logo }\end{array}$ & $\begin{array}{c}\text { Apple } \\
\text { Macbook }\end{array}$ & $\begin{array}{c}\text { Apple } \\
\text { Tree }\end{array}$ & $\begin{array}{c}\text { Green } \\
\text { Apple }\end{array}$ & $\begin{array}{c}\text { Red } \\
\text { Apple }\end{array}$ & \\
\cline { 3 - 8 } & 45 & 35 & 35 & 35 & 40 & 60 & 250 \\
\hline
\end{tabular}

Second dataset has 5 keywords which have 24 classes and the total numbers of images considered for analysis are 1000.In dataset 1 every keyword has 200 images. Every category has some number of images and all are stored in database.

Second dataset has 5 keywords which have 24 classes and the total numbers of images considered for analysis are 1000.In dataset 1 every keyword has 200 images. Every category has some number of images and all are stored in database.

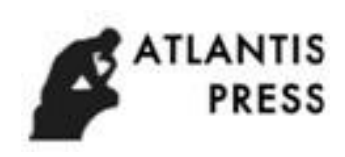




\subsection{Evaluation Parameters}

The most frequent and basic measures for information retrieval effectiveness are precision, recall and Mean Average Precision.

Precision $(\mathrm{P})$ is the fraction of retrieved images that are relevant as shown in Equation 3.

$$
\text { Precision }(P)=\frac{\text { No of relevant images retreived }}{\text { No of retreived images }}
$$

Recall (R) is the fraction of relevant images that are retrieved as shown in Equation 4.

$$
\operatorname{Recall}(R)=\frac{\text { No of relevant images retreived }}{\text { No of relevant images }}
$$

Average precision (AP) is calculated using Equation 5.

$$
A P=\left(\sum_{i=1}^{R} \frac{i}{\text { rank }_{i}}\right) / R
$$

Where $\mathrm{R}=$ number of relevant images for that query and $\left(\mathrm{i} / \mathrm{rank}_{\mathrm{i}}\right)=0$ if

Mean average precision (MAP) is calculated for a batch of queries using

Where $\mathrm{Q}=$ number of queries in a batch

$$
M A P=\left(\sum_{i=1}^{Q} A P_{i}\right) / Q
$$

\subsection{Working of the System}

User enters the query which undergoes stop word removal and stemming algorithm. For example, if the input query is "an apples" then the search query is "apple" where "an" is removed by stop word algorithm and " $\mathrm{s}$ " is removed from apples by applying stemming algorithm. The image search is done based on keyword apple.Fig.4 shows the output for query "an apples" where the users need to select a category.

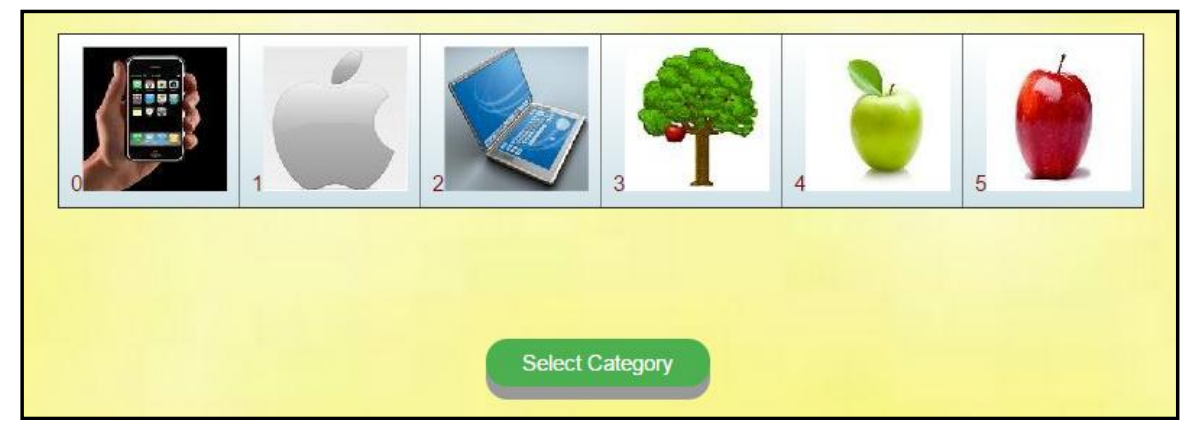

Fig. 4. Text based search result

If the user selects a particular category then images belonging to that category are displayed. This result will have relevant and irrelevant images for the selected category. User need to select an image for re-ranking which will act as query image. The query image can be stored in user log if it is needed by user otherwise can skip the option. If stored in user log it is used for further image retrieval.

User need to select keyword from user log and click search by log button then the images stored for the keyword is displayed as shown in Fig. 5. User log helps user to select the image without any hassle in less time. 


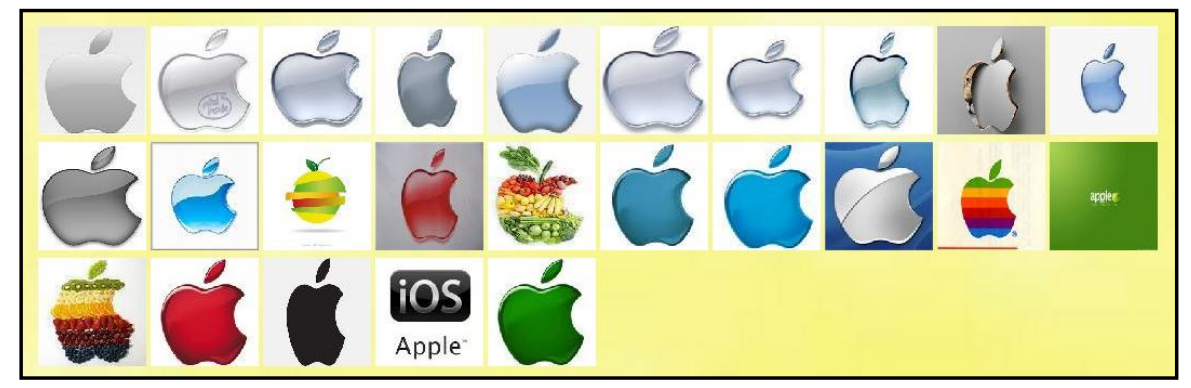

Fig.5.Re-ranked data using user log

\subsection{Experimental Results}

The Fig.6shows the Mean Average Precision for Dataset1. Query 1, 2 and 3 are different images which are considered as query image for calculating average precision.

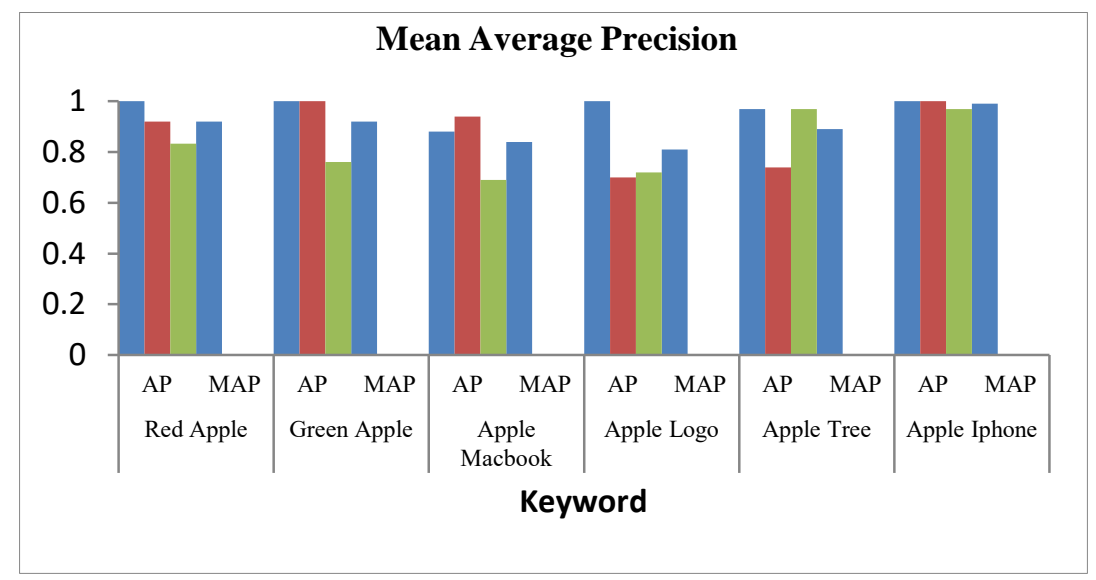

Fig.6.Mean average precision with respect to Dataset 1

The evaluation of precision with respect to image re-ranking with user log is shown in Fig.7.The precision rate for the proposed system is 93 better than the text based image retrieval system for Dataset 2.

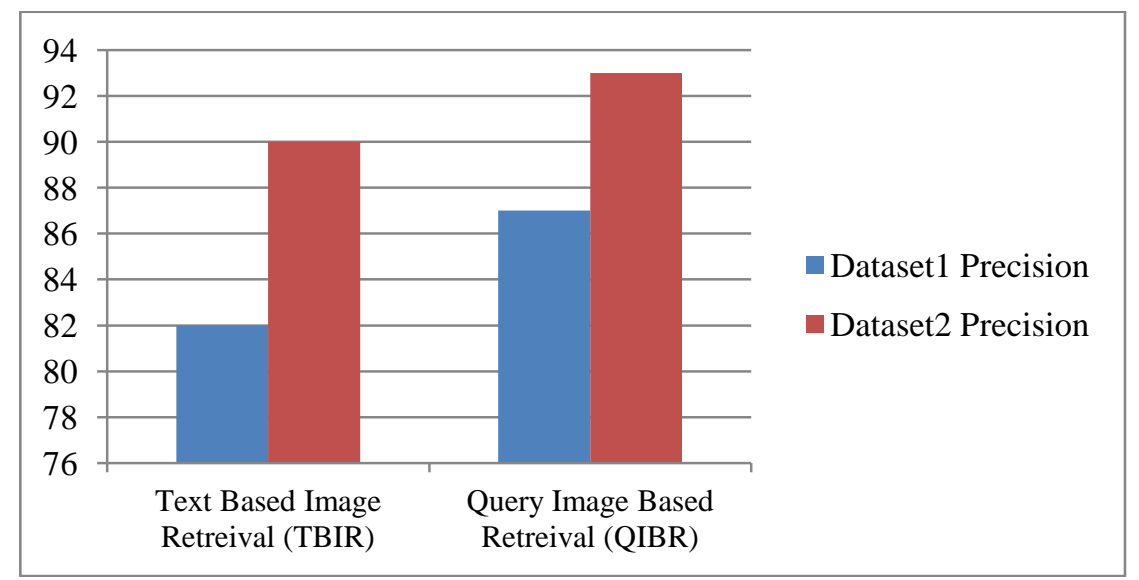

Fig.7.TBIR versus QIBR average precision for Dataset 1 and Dataset 2

Precision is improved when query image re-ranking is applied on the text based retrieved images. Fig.8 shows the precision of text based image retrieval (TBIR) and query image based retrieval (QIBR) for Dataset 2. 


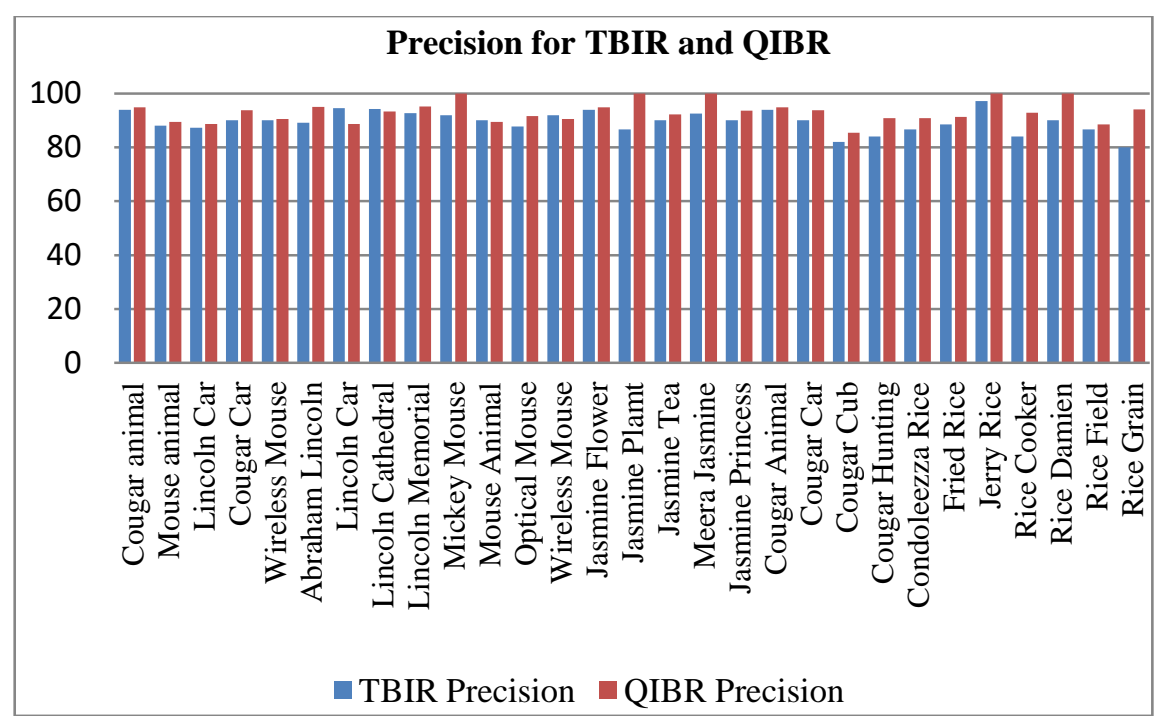

Fig.8.Performance comparison of TBIR and QIBR in term of Precision for Dataset 2

The performance of proposed system is also measured with respect to time factor where the $\mathrm{x}$-axis shows the image retrieval without log and the proposed with $\log$ and y-axis shows the time in seconds as shown in Fig.9.

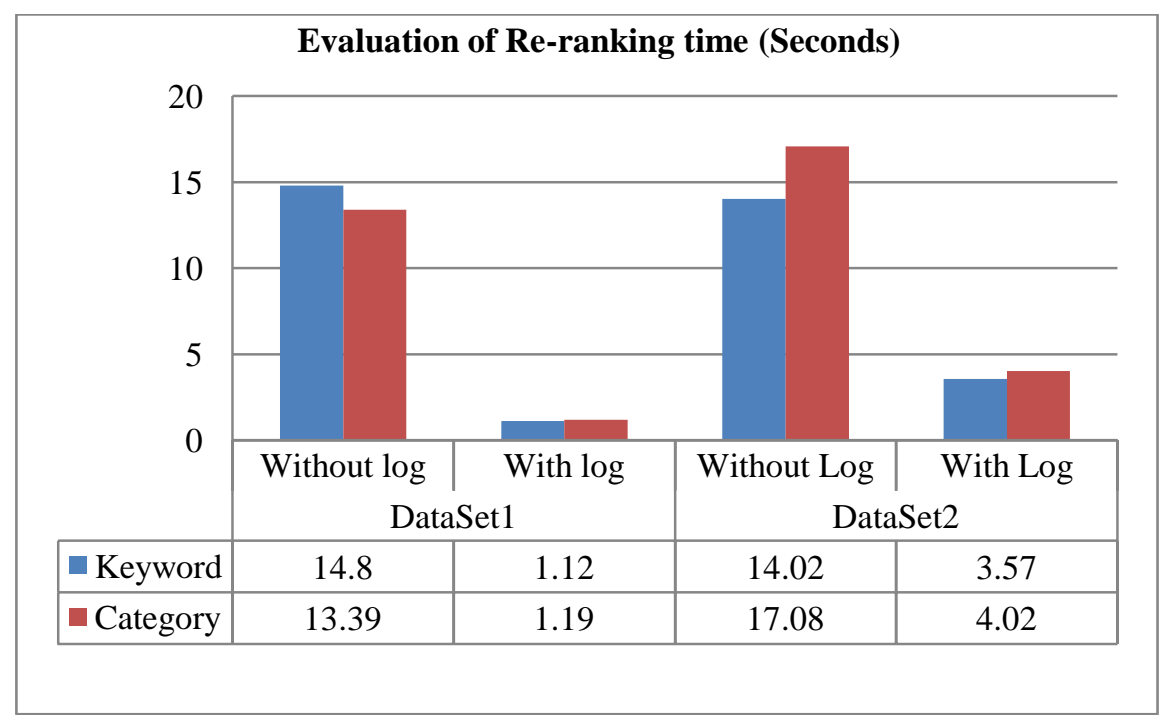

Fig.9.Performance comparison of log and without log in term of time

\section{Conclusions}

The proposed system allows the pre-primary school students to search images based on very simple keyword as query to understand the basic learning as part of play and learn. It has unique method to log the previous search keyword in database which makes it computationally superior and improves retrieved results. Initially images are retrieved based on given keyword. Then the system captures user's intention and retrieves images by comparing features of images which are stored offline. A set of keywords are expanded and their visual features are extracted at offline mode. As compared to the existing system, the proposed system improves the precision from $81 \%$ to $88 \%$ over Dataset 1 and $90 \%$ to $93 \%$ over Dataset 2 for TBIR and QIBR respectively. It also overcomes the drawback of user log statistics. The user log improves the performance of the system by reducing the image retrieval time. Though the system aims the academic curriculum of pre-primary school students, it can play a significant role in the field of medical for analyzing anatomy of human body. 


\section{References}

[1] Feng Jing, Changhu Wang, Yuhuan Yao, Kefeng Deng, Lei Zhang, Wei-Ying Ma: Web Image Search Results Clustering,Proceedings of the 14th annual ACM international conference on Multimedia, pp. 377-384 (2006)

[2] Ying Liua, Dengsheng Zhanga, Guojun Lua, Wei-Ying Ma: A Survey of content-based image retrieval with high-level semantics, Science Direct, pp. 262-282 (April 2006)

[3] Xinmei Tian, Dacheng Tao, Xian-Sheng Hua, Xiuqing Wu: Active Reranking for Web Image Search. IEEE Transactions on image processing Vol. 19, No. 3, pp. 805-820 (March 2010)

[4] Lixin Duan, Wen Li, Tsang.I.W: Improving Web Image Search by Bag-Based Reranking. IEEE Transaction on image processing, Vol. 20, No. 11, pp. 3280-3290(November 2011)

[5] Xiaoou Tang, Ke Liu, Jingyu Cui, Fang Wen, Xiaogang Wang: IntentSearch: Capturing User Intention for OneClick Internet Image Search. IEEE Transactions on pattern analysis and machine intelligence, Vol. 34, No. 7, pp. 1342 1353 (July 2012)

[6] Xiaogang Wang, Ke Liu, Xiaoou Tang: Web Image Re-Ranking Using QuerySpecific Semantic Signature. IEEE Transactions on Pattern Analysis and Machine Intelligence, Vol. 36, No. 4, pp. 810-823 (April 2014)

[7] Jalil Abbas, Salman Qadr, Muhammad Idrees, Sarfraz Awan, Naeem Akhtar Khan: Frame Work for Content Based Image Retrieval (Textual Based) System. Journal of American Science, vol. 6, no. 9, pp. 704-707 (2010)

[8] Jun Huang, Xiaokang Yang, Xiangzhong Fang, Weiyao Lin, Rui Zhang: Integrating visual saliency and consistency for re-ranking image search results. Multimedia, IEEE Transactions, vol. 13, no. 4, pp. 653-661 (2011)

[9] N. Rasiwasia, P.J. Moreno, N. Vasconcelos: Bridging the Gap: Query by Semantic Example. IEEE Trans. Multimedia, vol.9, no. 5, pp. 923-938 (Aug 2007)

[10] N Tao Mei, Yong Rui, Shipeng Li, Qi Tian: Multimedia Search Reranking: A $\quad$ Literature Survey ACM Journal. Vol. 2, No. 3, Article 1 (May 2012)

[11] W. $\quad$ B. Frakes: Software Engineering Guild, Sterling, $\quad$ VA 22170.http://dns.uls.cl/ ej/daa_08/Algoritmos/books/book5/chap08.htm (2016)

[12] Jing-Ming Guo, Heri Prasetyo: Content-Based Image Retrieval Using Features Extracted From Halftoning-Based Block Truncation Coding. IEEE Transactions on image processing, Vol. 24, No. 243, pp. 1010-1024 (March 2015)

[13] Veronica S. Moertini: Introduction to five data clustering algorithms. INTEGRAL, Vol. 7, No. 2, pp. 87-96(October 2002)

[14] Annesha Malakar, Joydeep Mukherjee2: Image Clustering using Color Moments, Histogram, Edge and K-means Clustering. International Journal of Science and Research (IJSR), India Online ISSN: 2319-7064, Volume 2 Issue 1, pp. 532-537 (January 2013) 